\title{
Früherkennung und zivile Krisenprävention: Wissenschaftlich unmöglich, politisch überflüssig?
}

Der Aktionsplan „Zivile Krisenprävention, Konfliktlösung und Friedenskonsolidierung“ feierte kürzlich von der Öffentlichkeit nahezu unbemerkt seinen zehnten Geburtstag. Die rot-grüne Bundesregierung hob ihn im Mai 2004, fünf Jahre nach ihrer Beteiligung am Kosovokrieg, aus der Taufe. Sie hoffte darauf, durch frühzeitiges Erkennen krisenhafter Entwicklungen künftig gewaltsamer Konfliktaustragung effektiver vorbeugen zu können. Dies trug ihr insbesondere aus Kreisen der Friedensbewegung wie der Friedensforschung viel Lob ein. Dennoch ließ Kritik nicht lange auf sich warten: Der Plan komme mit seiner Auflistung von 161 Maßnahmen über einen sogar militärische Mittel umfassenden Kessel Buntes nicht hinaus. Neue Etiketten verschleierten nur den ausbleibenden Politikwechsel. Die Einbeziehung zivilgesellschaftlicher Kräfte verlaufe unbefriedigend. Eine Prämisse blieb jedoch unstrittig: Früherkennung und zivile Krisenprävention sind sowohl wissenschaftlich möglich als auch politisch sinnvoll. Aber stimmt das überhaupt? Die Vielzahl explosiver Konstellationen spricht auf den ersten Blick dagegen. Erinnert sei an die Eskalation des Konflikts in der Ukraine, der bereits verschüttet geglaubte Gräben zwischen dem Westen und Russland wieder aufriss. Nach Einschätzung hiesiger Politiker handelt es sich sogar um die größte Krise seit Ende des Kalten Kriegs. Doch wo liegt das Problem? Beruht bereits die Vorstellung, inner- wie zwischenstaatliche Prozesse durch permanente Beobachtung und Analyse prognostizieren und durch geschicktes Eingreifen in friedlichen Bahnen halten zu können, auf einem Trugschluss? Oder folgt Politik ihrer eigenen Logik, die sich eben nicht systematisch an den Erfordernissen ziviler Krisenbearbeitung orientiert? Möglicherweise fehlt aber auch nur ein Transmissionsriemen, der die Befunde der Früherkennung an der richtigen Stelle in den politischen Prozess einspeist? S+F hat Experten bzw. Expertinnen mit unterschiedlichem beruflichem Hintergrund gebeten, den Zusammenhang von Früherkennung und ziviler Krisenprävention aus ihrer Sicht zu beleuchten.

Sabine Jaberg

\section{Frühwarnsysteme schüren in der Politik trügerische Hoffnungen}

\section{Jan Pospisil}

Sind Krisenfrühwarnsysteme eine sinnvolle Einrichtung? Allein diese Frage mag auf den ersten Blick Verwunderung auslösen. Wie könnten sie denn nicht sinnvoll sein? Ist es nicht unabdingbar, so früh und umfassend wie möglich über potenzielle Krisen informiert zu sein, um entsprechende präventive Aktionen ergreifen zu können? Und selbst im Falle eines Scheiterns von Prävention gilt schließlich, dass auch reaktive Maßnahmen im Krisenfall über eine kritische Zeitkomponente verfügen. Aufbauend auf einem Forschungsprojekt des Österreichischen Instituts für Internationale Politik, das bestehende Konfliktfrühwarnsysteme sowie Ansätze der standardisierten Konfliktanalyse qualitativ miteinander vergleicht, ${ }^{1}$ möchte ich die Sinnfrage dennoch stellen.

Zu diesem Zweck ist es zunächst notwendig, eine Einschränkung und eine grundsätzliche Klärung vorzunehmen. Ich beziehe mich ausschließlich auf Frühwarnsysteme, die auf bewaffnete Konflikte ausgerichtet sind. Warum ist diese Einschränkung so wichtig? Weil es sich bei bewaffneten Konflikten um soziale Prozesse handelt. Und soziale Prozesse verlaufen insofern in grundlegend anderen Bahnen als etwa Naturkatastrophen, als sie keinerlei Automatismen unterliegen, sondern von bewussten Entscheidungen der involvierten Akteure abhängen. Dementsprechend gibt es auch keine Möglichkeit einer exakten Vorhersage. Die beste Sozialwissenschaft kann keine unumstößliche

1 Das Projekt führte ich gemeinsam mit Kollegen in den Jahren 2009 und 2010 durch. Es wurde durch Fördergelder des Jubiläumsfonds der Oesterreichischen Nationalbank unterstützt (Projektnummer: 13.101).
Prognose gewährleisten. So trivial diese Einschränkung klingen mag, so sehr wird ihre Bedeutung nach wie vor unterschätzt, was sich insbesondere anhand der oftmals naiven Erwartungshaltungen gegenüber Frühwarnprozessen ablesen lässt.

Wie lautet nun die Ausgangshypothese der Befürworter und Befürworterinnen von Konfliktfrühwarnung? Sie basiert auf drei Säulen: (1) Es gibt unzureichende Informationen aus jenen fragilen Regionen, in denen ein bewaffneter Konflikt auftreten könnte; (2) es existiert ein Defizit sowohl in der Sichtung als auch der systematischen, prognoseorientierten Aufarbeitung derartiger Information; und (3) es besteht ein Mangel bei der Verbreitung dieser Information, was schließlich in ungenügende oder gar ausbleibende Early Action mündet. Wären also - nach dieser Logik - den maßgeblichen Entscheidungsträgern die Fakten bekannt und wären sie darüber hinaus von deren Richtigkeit und Bedeutung überzeugt, würde neben dem Wissen um sinnvolle Optionen auch der politische Handlungsdruck zunehmen. Es gäbe zwar immer noch keinen Automatismus, aber eine Early Action würde viel wahrscheinlicher.

Sicherlich sind diese drei Annahmen nicht generell zurückzuweisen, aber jede für sich ist in ihrer Substanz hinterfragbar: Nichtwissen oder zu spät eingetroffene Erkenntnisse waren in den seltensten Fällen der Grund für ein Nichteinschreiten externer Akteure. Selbst bei vermeintlich überraschenden Ereignissen lagen entsprechende Informationen, Berichte und Analysen vor. Als nur ein Beispiel seien die vermeintlich gänzlich unerwarteten Gewaltausbrüche im Zuge der kenianischen Wahlen 2007/2008 erwähnt. Auch hier gab es analytisches Material, das die problematische ethnopolitische Aufladung der Situation aufzeigt. Nur wurde diesen Informationen offenbar nicht die notwendige Glaubwürdigkeit geschenkt bzw. die entsprechende politische Bedeutung beigemessen. 
Wenn ein grundlegendes Defizit zu konstatieren ist, dann auf epistemologischer Ebene: Die Politik steht mittels qualitativer Methodik gewonnenen Erkenntnissen nach wie vor skeptisch gegenüber. Quantitativen Datenreihen hingegen billigt sie ein viel höheres Maß an Glaubwürdigkeit und Relevanz zu, was einen strategischen Vorteil nicht nur bei der Verbreitung der Befunde, sondern auch bei der Erörterung erforderlicher Entscheidungen bewirkt. Dieser Vertrauensvorschuss gegenüber statistisch-quantitativem Material ist gerade im Bereich der Konfliktanalyse keineswegs per se gerechtfertigt; die Güte der Ergebnisse lässt sich nicht anhand der angewandten Methode a priori festmachen. Aber auch die quantitative Datenlage ist in Zeiten einer funktionierenden Globalüberwachung durch Satellitensysteme keineswegs als mangelhaft zu bewerten. Frühwarnung ist hier nicht nur problemlos möglich, sie findet auch statt - nicht nur bei politischen Entscheidungsträgern und in militärischen Lagezentren. Vielmehr kommen die Informationen durch den immer engeren Austausch zwischen Staaten- und Gesellschaftswelt auch bei Nichtregierungsorganisationen an, wobei sich deren eigene Lagekenntnis ohnehin zumeist auf dem aktuellsten Stand befindet.

Wenn nun die Ausgangshypothese fragwürdig ist, warum dennoch dieses Mantra von der Notwendigkeit und dem weiteren Auf- bzw. Ausbau von Konfliktfrühwarnsystemen? Ich vermute, dass diese Hypothese für alle Beteiligten einfach zu angenehm war, um sie fallen zu lassen - wobei natürlich angemerkt werden muss, dass der Hype um Konfliktfrühwarnung in den letzten Jahren merklich nachgelassen hat. Warum angenehm? Die Anerkennung, dass Krisenprävention aufgrund technischer Defizite - eben einer mangelnden Frühwarnung - nicht so funktioniert wie sie funktionieren sollte, bietet etliche Vorteile. Für die im Bereich der Early Action politisch Verantwortlichen bedeutet es keinerlei Risiko, Informationskapazitäten auszubauen. Und billiger als jedes konkrete Einschreiten ist dies allemal. Damit werden Frühwarnsysteme zu einer kostengünstigen Ersatzhandlung. Außerdem hilft die Hypothese sehr gut dabei, die politische Verantwortlichkeit für gescheiterte Krisenprävention mit vorgeschobenen technischen Mängeln zu verschleiern. Aber auch für diejenigen, die Frühwarnsysteme erforschen, konzipieren und umsetzen, besitzt das Mantra Relevanz: Ohne Zweifel glauben sie daran, etwas Richtiges und Wichtiges zu tun. All dies geht mit dem angenehmen Nebeneffekt einher, die eigene Stelle ressourcentechnisch abzusichern.

Vor dem Hintergrund dieser Allianz besteht ein veritables Risiko zur weiteren Aufrechterhaltung eines Cargo-Kultes: Zwar wird ohne Zweifel korrekt entlang der Ausgangshypothesen gearbeitet (Defizite in Information, Verarbeitung, Verbreitung) - die letztendliche Early Action fand und findet dennoch nicht statt, zumindest nicht im Sinne des angestrebten Automatismus, der aufgrund gegebener Informationslagen ausgelöst würde. Es gibt keine technische Lösung politischer Entscheidungen, und Krisenprävention wird immer eine politische Entscheidung bleiben.

Für Wissenschaft und Forschung zeitigt diese Einsicht auf zwei Ebenen Konsequenzen: Erstens bedeutet die hier dargelegte Skepsis gegenüber Konfliktfrühwarnsystemen keineswegs, dass unser Wissen über bewaffnete Konflikte auf einem ausreichenden Stand wäre. Im Gegenteil: Wir wissen nach wie vor viel zu wenig über ihre Ursachen, ihre Verläufe und vor allem ihre Beendigung. Alle Versuche, dieses Wissen mittels Konfliktzyklen oder ähnlichen Instrumentarien zu systematisieren und in einfacher Weise handhabbar zu machen, scheitern immer wieder an komplexen Realitäten. Ein vertieftes, kontextualisiertes Verständnis konkreter Konstellationen kann ebenso wenig durch Frühwarnsysteme ersetzt werden wie eine tiefergehende analytische und konzeptionelle Auseinandersetzung mit strukturellen Konfliktursachen. Zweitens besitzen wir nach wie vor zu wenige Kenntnisse über die sinnvolle Ausgestaltung von Krisenprävention als gesamtstaatlicher, aber auch gesamtgesellschaftlicher Aufgabe. Zwar gelten Entscheidungsabläufe und Beschränkungen von Policy-Prozessen als gut erforscht. Aber die speziell im Bereich des international orientierten politischen Handelns zunehmende Fokussierung auf Ansätze wie einen Whole-of-Government-Approach bedeuten zunächst eine Multiplikation der zu beantwortenden Fragen: Welche Instrumente können sinnvoll miteinander agieren? Wie lassen sich Kommunikationsprozesse optimieren? Wie müssten staatliche und nichtstaatliche Akteure im Bereich der Krisenprävention kooperieren? Und schließlich: Welche Konzepte und Zielsetzungen sollen einem solchen Engagement zugrunde liegen?

Gegenwärtig erscheinen mir diese zwei Problemkomplexe in der wissenschaftlichen Auseinandersetzung mit der Prävention bewaffneter Konflikte nicht nur entscheidender, sondern mit Blick auf die betroffenen Regionen dringlicher als die Frage nach Konfliktfrühwarnsystemen. Deren implizite Versprechung, politische Probleme einer technischen Lösung zuzuführen, hat sich nicht erfüllt.

Dr. Jan Pospisil ist Senior Researcher am Österreichischen Institut für Internationale Politik (oiip) und Lehrbeauftragter an der Universität Wien.

\section{Das Frühwarnsystem der OSZE hilft ziviler Krisenprävention}

\section{Claus Neukirch}

Auf ihrem Ministerratstreffen am 6./7. Dezember 2011 in Wilna beschlossen die Teilnehmerstaaten der Organisation für Sicherheit und Zusammenarbeit in Europa (OSZE), deren Kapazitäten im Bereich Frühwarnung zu stärken. Sie beauftragten den Generalsekretär, u.a. „dafür zu sorgen, dass das Konfliktverhütungszentrum (KVZ) des Sekretariats die Rolle und Funktionen einer Schaltstelle für systematische Sammlung, Zusammenführung, Analyse und Bewertung der maßgeblichen Frühwarnsignale verschiedenster Herkunft für die gesamte Organisation übernimmt“. ${ }^{2}$ Die Ministerratsentscheidung von Wilna erteilt dem Generalsekretär zudem das Mandat, „in Absprache mit dem Vorsitz für Frühwarnung an die Teilnehmerstaaten zu sorgen und zu diesem Zweck dem Ständigen Rat jeden Fall neuer oder erneut auftretender Spannungen oder

2 Beschluss Nr. 3/11 über Elemente des Konfliktzyklus im Zusammenhang mit der Verstärkung der Fähigkeiten der OSZE in den Bereichen Frühwarnung, Frühzeitiges Handeln, Dialogerleichterung und Mediationsunterstützung sowie Konfliktnachsorge,(MC.DEC/3/11/Corr.1 vom 7. Dezember 2011), http://www.osce.org/de/mc/88841?download=true am 25.2.2014. 
Konflikte im OSZE-Raum zur Kenntnis zu bringen“" sowie ihm nach Rücksprache mit dem betreffenden Teilnehmerstaat (oder Staaten) Optionen für zeitnahe und wirkungsvolle Reaktionen vorzuschlagen. Diese Frühwarnfunktion des Generalsekretärs ergänzt die in den jeweiligen Mandaten aller maßgeblichen Durchführungsorgane der OSZE bereits enthaltenen Frühwarnfunktionen, wobei dem Hohen Kommissar für nationale Minderheiten (HKNM) besondere Bedeutung zukommt.

In den vergangenen zwei Jahren hat das KVZ die Wilnaer Entscheidung durch die Ausarbeitung interner Richtlinien zur Frühwarnung, die Entwicklung von standardisierten Vorlagen zur Konfliktanalyse und Berichterstattung sowie den Aufbau eines Netzwerks von focal points operativ ausgestaltet. Zwischen Januar 2012 und Januar 2014 hat der Generalsekretär in seinen Berichten an den Ständigen Rat insgesamt zwölfmal auf erhöhte und eskalierende Spannungen hingewiesen, dabei allerdings nur einmal explizit eine „Frühwarnung“ ausgesprochen. In zwei Fällen ist es (dennoch) zu einer - wenn auch kurzzeitigen - gewaltsamen Eskalation mit mehreren Toten gekommen.

Aus den bisherigen Erfahrungen lassen sich einige Schlussfolgerungen für die praktische Umsetzung von Frühwarnkonzepten ziehen: Die Frage, ob Frühwarnung überhaupt möglich ist, lässt sich in der Praxis mit einem klaren „Jein!“ beantworten. Auf der einen Seite schärft eine gute Analyse, die strukturelle Konfliktlinien ebenso wie potenzielle Auslösefaktoren aufzeigt, in jedem Fall das Verständnis von Entscheidungsträgern für die jeweilige Situation und deren Eskalationspotenzial. Ein systematisches Frühwarnsystem wie das der OSZE führt zudem dazu, dass Mitarbeiterinnen und Mitarbeiter in den Feldmissionen der Organisation, wie auch in den Institutionen und im Sekretariat, potenziell krisenhaften Entwicklungen generell mehr Aufmerksamkeit schenken. Da jede interne Frühwarn-Berichterstattung automatisch denkbare OSZE-Maßnahmen mitbehandelt, werden auch mögliche Gewaltpräventions- und Krisenreaktionsmaßnahmen früher und systematischer diskutiert. Die organisationsinterne Frühwarn- und Krisenpräventionskultur wird somit gestärkt.

Auf der anderen Seite ist die technische und personelle Kapazität, die Organisationen wie der OSZE für eine umfassende Frühwarnung zur Verfügung steht, in Zeiten strikter Haushaltsführung begrenzt. Ein alle potenzielle Konfliktfaktoren erfassendes und ständig aktualisiertes Frühwarnsystem lässt sich daher in der Realität nur äußerst schwer aufbauen. Selbst wenn dies geschähe - eine exakte Vorhersage krisenhafter $\mathrm{Zu}$ spitzungen ist auch mit dem besten Frühwarnsystem nicht zu bewerkstelligen. So kann eine konfliktträchtige Situation lange stabil bleiben und dennoch durch einen nicht vorhersehbaren „Auslöser“ - wie etwa Naturkatastrophe, Unfall, Mord - schnell in Gewalt umschlagen. Ein gutes Frühwarnsystem wird zwar die strukturellen Konfliktursachen herausarbeiten und präventive Maßnahmen empfehlen, es wird ihm aber nur schwer möglich sein, einen Gewaltausbruch oder gar dessen Eintrittszeitpunkt mit Bestimmtheit zu prognostizieren. Auch werden Frühwarnsysteme nicht in der Lage sein, mit Gewissheit vorherzusagen, ob und wann Entscheidungsträger auf den Einsatz von Gewalt setzen. Zudem kann das in den letzten Jahren erkennbare Mobilisierungspotenzial sozialer Netzwerke Dynamiken freisetzen,

3 Ebd. die von bestehenden Frühwarnsystemen nur unzureichend abgedeckt werden können. Effektive Frühwarnung ist somit nur eingeschränkt möglich. Potenziell krisenhafte Entwicklungen können zwar in den meisten Fällen antizipiert, als Szenarien skizziert und mit Wahrscheinlichkeitsprognosen versehen werden, die Genauigkeit dieser Einschätzungen wird jedoch begrenzt bleiben. Das Risiko, von dynamischen Entwicklungen überrascht und überrollt zu werden, lässt sich daher nur minimieren, aber nicht vollkommen ausschließen.

Unabhängig von der statistischen Treffergenauigkeit von Frühwarnung ist der Aufbau eines effektiven Frühwarnsystems für die zivile Krisenprävention von entscheidender Bedeutung. Auch in einem eher auf „späte“ Frühwarnung konzentrierten System, wie jenem der OSZE, hilft die systematische Auseinandersetzung mit strukturellen Konfliktursachen und potenziellen Auslösefaktoren dabei, frühzeitig präventive Maßnahmen zu entwerfen und gegebenenfalls umzusetzen. Dennoch ist effektive Frühwarnung kein Erfolgsgarant. Die eigentliche Herausforderung liegt eben nicht auf der Ebene der Frühwarnung, sondern auf der Ebene des frühzeitigen präventiven Handelns. Hier kommt allerdings die ganze Fülle operativer, politischer und oft auch persönlicher Faktoren ins Spiel. Sie entscheiden darüber, ob präventive Maßnahmen rechtzeitig und zielgerichtet ergriffen werden. Nicht zuletzt deshalb verbindet die Entscheidung des Ministerrats von Wilna die Erhöhung der Kapazitäten im Bereich der Frühwarnung mit Maßnahmen zur Stärkung der OSZE in den Bereichen Vermittlung, Dialogerleichterung und frühzeitiges präventives Handeln.

Welche Konsequenzen ergeben sich aus diesem Befund für Frühwarnung und Krisenprävention in der Praxis? Die wichtigste Schlussfolgerung lautet: Akteure, die krisenpräventiv wirken wollen, sollten trotz der inhärenten Beschränkungen von Frühwarnsystemen entsprechende Kapazitäten aufbauen und ernsthaft unterhalten, da mit ihrer Hilfe die operative Planung und Vorbereitung krisenpräventiver Maßnahmen entscheidend vorverlegt werden kann. Um dies zu erreichen ist es erforderlich, dass ein an die Bedürfnisse des Akteurs angepasstes Frühwarnsystem nicht nur auf dem Papier existiert, sondern fest in interne Arbeits- und Planungsabläufe eingebunden wird. Um diesen - durchaus längerfristigen Prozess - zu erleichtern, gilt es, die Balance zwischen einem idealen, akademisch ausgereiften System und den aus realen Gegebenheiten resultierenden Beschränkungen zu wahren. Nicht alles, was technisch machbar und akademisch wünschenswert wäre, lässt sich in Anbetracht des Profils und der Arbeitsbelastung von Mitarbeiterinnen und Mitarbeitern, finanziellen Ressourcen und technischen Möglichkeiten sowie Mandat und Ausrichtung der entsprechenden Einrichtung auch umsetzen. Wird diese Balance nicht gewahrt, lässt sich das Frühwarnsystem entweder nicht wie erforderlich integrieren oder generiert keine brauchbaren Informationen.

Abschließend gilt es noch zwei Punkte zu bedenken. Auch eine formelle Frühwarnung, die etwa der Generalsekretär an den Ständigen Rat der OSZE richtet, kann bereits frühzeitiges Handeln darstellen, da dies zumindest implizit einen Aufruf an die Teilnehmerstaaten darstellt, entsprechenden Entwicklungen mehr Aufmerksamkeit zu schenken. Idealerweise setzt eine Frühwarnung dieser Art Mechanismen in Gang, die einer weiteren Eskalation entgegenwirken. Eine erfolgte Frühwarnung 
ist mithin bereits eine Form frühzeitigen präventiven Handelns, um auf diplomatischer Ebene krisenpräventive Schritte anzuregen. Würde sie sich im Nachhinein als „falsch“ erweisen, da die Eskalation aufgrund präventiver Maßnahmen ausbleibt, dann hätte sie einen wichtigen Beitrag zur erfolgreichen Krisenprävention geleistet. Man sollte daher bei aller Kritik an der Ungenauigkeit von Frühwarnsystemen nicht das Kind mit dem Bade ausschütten, sondern versuchen, das unter den jeweiligen Bedingungen bestmögliche System aufbauen.

Dr. Claus Neukirch ist Stellvertretender Leiter des Konfliktverhütungszentrums der Organisation für Sicherheit und Zusammenarbeit in Europa (OSZE). Der Beitrag gibt ausschließlich die persönliche Meinung des Verfassers wieder.

\section{Frühwarnung und zivile Krisenprävention müssen besser miteinander verzahnt werden}

\section{Angelika Spelten}

Ägypten, Syrien, Mali und nicht zuletzt die Entwicklungen in der Ukraine machen deutlich: Frühwarnung und Krisenprävention haben in der Vergangenheit öfter versagt als genutzt. Der Anspruch, der in den 1990er Jahren an Prävention formuliert wurde, eine gewaltsame Zuspitzung politischer Krisen zu verhindern, konnte nicht eingelöst werden.

Die Stiftung Wissenschaft und Politik (SWP) legte jüngst in einer Studie zur Außenpolitikevaluation einen Überblick über wissenschaftliche Einschätzungen zu den Ansätzen und dem Anspruch von Krisenprävention wie ziviler Konfliktbearbeitung vor. Ihre grundlegende Kritik lautet, dass der Präventionsgedanke von linearen Ursache-Wirkungs-Zusammenhängen ausgeht, die den realen Dynamiken nicht entsprechen. Er setze zudem ein hohes Maß an Wissen über die Beeinflussung komplexer sozialer Prozesse voraus, das in der Wissenschaft so jedoch nicht vorhanden sei. ${ }^{4}$ Ergo: Das ganze Politikfeld stehe auf tönernen Füßen und jage einer Vision nach, der eine solide Grundlage für die Umsetzung fehle.

Aus der Praxis-Perspektive betrachtet ist diese Diagnose so richtig wie wenig hilfreich. Sie befindet sich in gewissem Widerspruch zur Erfahrung, dass Frühwarnung und zivile Krisenprävention in der Realität durchaus stattfinden, wenngleich die Ergebnisse in vielen, aber eben nicht in allen Fällen enttäuschen.

Daher gibt es auch wissenschaftliche Stimmen, die der Überzeugung sind, die Erfolgsaussichten für Friedensstabilisierung seien umso größer, je früher im Verlauf einer Krisenentwicklung Gegenstrategien einsetzen. Dieses Paradigma hat sich in Fachkreisen und Medien etabliert. Wenn Prävention versagt und Gewalt eskaliert, stehen daher schnell die Fragen im Raum: Waren internationale Stellen auf diese Entwicklung nicht vorbereitet? Hat also die Frühwarnung versagt? Man unterstellt damit, dass eine längere Vorwarnzeit immer auch zu mehr Erfolg bei ziviler Krisenprävention führt. Betrachtet man aber die

4 Peter Rudolf/Sascha Lohmann, Außenpolitikevaluation im Aktionsfeld Krisenprävention und Friedensaufbau, SWP-Studie, Berlin Oktober 2013. jüngsten Gewaltentwicklungen in verschiedenen Regionen der Welt, ergibt sich ein gemischtes Bild über den Zusammenhang von Frühwarnung und frühzeitigem Handeln.

Für die Entwicklungen des „Arabischen Frühlings“ fehlte tatsächlich jegliche Frühwarnung. Zwar waren die Missstände schon lange bekannt, die den Boden für die revolutionären Aufstände bereiteten, aber kaum ein Frühwarn- oder Regionalexperte sah voraus, dass Ende 2010/Anfang 2011 ein derart hohes Eskalationsrisiko bestand. Die internationale Gemeinschaft reagierte dann zwar relativ schnell, zeigte sich jedoch im Zuge der weiteren Eskalation in Libyen und Syrien recht hilflos. Zivile Handlungsoptionen wie die Sperrung von Bankkonten oder Reisebeschränkungen für bestimmte Personengruppen wurden erst spät und noch dazu zögerlich ergriffen, während der Reflex militärischer Intervention zumindest im Falle Libyens vergleichsweise schnell und konzeptionslos einsetzte.

Das Risiko eines neuen Bürgerkriegs im Südsudan war hingegen absehbar. Seit der Verabschiedung der Übergangsverfassung im Juli 2011 nahmen die Spannungen zwischen den Volksgruppen der Dinka und Nuer bedrohlich zu, worauf Regionalexperten auch hinwiesen. Dennoch gab es keine konkrete Präventionsstrategie von internationaler Seite. Erst mit Ausbruch von Kampfhandlungen im Januar 2014 setzten sich europäische Staaten und die USA für eine Verhandlungslösung ein, die jedoch scheiterte.

Eine andere Konstellation bestand im Falle Kenias. Erst relativ spät, ca. sechs Monate vor dem Wahltermin im März 2013, alarmierten Regionalexperten und die International Crisis Group, dass auch dieser Urnengang, wie bereits die Wahl 2007, in Gewalt enden könnte. Für dieses Risiko waren die vor Ort tätigen Entwicklungsorganisationen jedoch bereits seit Langem sensibilisiert. Nach den Unruhen von 2007/2008 begann ein von der internationalen Gemeinschaft begleiteter tiefgreifender Reformprozess. In dessen Verlauf zeigte sich mehrfach, dass die alten Konkurrenzverhältnisse und Konfliktmuster weiterhin wirkten. Dass der nächste Wahltermin daher eine äußerst kritische Zäsur sein würde, war den meisten Programmverantwortlichen seit Beginn des Reformprozesses bewusst.

Diese Beispiele illustrieren zweierlei: Erstens ist die Krisenvorwarnzeit in bestimmten Kontexten deutlich kürzer geworden. Gewaltagitation und ihre logistische Vorbereitung erfolgen heutzutage mit modernen Kommunikationsmedien. Stimmungsmache gegen ethnische oder religiöse Minderheiten, den politischen Gegner oder gezielte Falschinterpretationen von Gesetzesinitiativen können so in kurzer Zeit mit wenig Aufwand das gesellschaftliche Klima gefährlich aufheizen und politischen Dynamiken eine gewaltsame Richtung geben. Im Vergleich zu den 1990er Jahren verlaufen solche Prozesse aber nicht nur deutlich schneller, sondern sie bleiben externen Beobachtern und Frühwarnexperten oft verborgen. Die klassischen indikatorbasierten Frühwarnsysteme, die ein- bis zweimal jährlich ein Situationsbild erstellen, können diese Entwicklungen kaum erfassen. Bei den politischen Entscheidungsträgern möchte man dennoch den Bedarf an Orientierungshilfe unterstellen, die dazu beiträgt, dass angemessene Handlungsoptionen schneller identifiziert und ihre Wirkungsweise besser eingeschätzt werden können. Zweitens sind internationale und nationale Institutionen mittlerweile für viele mittelfristige Krisenverläufe sensibilisiert. Hier besteht offenbar 
kaum Bedarf an weiterer Frühwarnung. Das bedeutet jedoch nicht, dass auch frühzeitig deeskalierende Strategien entwickelt und umgesetzt würden. Hier zeigt sich eher ein nach wie vor eklatanter Mangel an angemessenen Handlungsoptionen.

Bislang lässt sich also kaum ein systematischer Zusammenhang zwischen Frühwarnung und Krisenreaktionsansätzen erkennen. Die Schlüsselfrage lautet daher nicht, ob Frühwarnung generell möglich ist, sondern wie sie gestaltet sein muss, um die Erfolgsaussichten ziviler Krisenprävention zu verbessern. Neuere Frühwarninstrumente nutzen zwar auch die Vorteile von Internet und Facebook, um Informationen und Analysen möglichst zeitnah bereitzustellen. Das führt allerdings zunächst nur zu einem deutlichen Aufwuchs der Informations- und Datenmenge. Regierungsstellen, Botschaften, internationale Organisationen und Nichtregierungsorganisationen, die sich mit präventiven Maßnahmen engagieren möchten, zeigen sich mit der Auswertung und Interpretation dieser Informationsmengen in vielen Fällen überfordert.

Es ist daher dringend erforderlich, Frühwarnung so zu gestalten, dass sie zum einen neben langfristigen Prozessen auch kurzfristige Zäsuren, potenzielle Kumulationspunkte und sogenannte „Trigger-Faktoren“ erfasst und die von ihnen ausgehende Dynamik beobachtet. Zum anderen müssen die Ergebnisse für politische Entscheidungsträger schneller zur Verfügung stehen. Als mögliche Methode bietet sich die systemische Szenarienanalyse an. Im Idealfall identifizieren Vertreter aus der jeweiligen Krisenregion gemeinsam mit westlichen Partnern, welche lokalen Faktoren und Akteure aktuell den größten Einfluss auf die weitere Stabilisierung oder Eskalation der Krise haben und wie sich diese Faktoren gegenseitig beeinflussen. Diese Analyse würde in angemessenen zeitlichen Abständen aktualisiert und mit der Frage verbunden, durch welche Maßnahmen auch externe Akteure Stabilisierungstendenzen unterstützen können. Aber sogar derart verbesserte Verfahren wären keine Erfolgsgaranten. Wo liegen mögliche Fallstricke?

Frühwarnung bleibt wirkungslos, wenn präventives Handeln ausbleibt. Es besteht daher auch dringender Bedarf, die Kapazitäten zur Entwicklung konkreter und wirkungsmächtiger Handlungsoptionen auszubauen. Für Bürgerkriege - wie beispielsweise in der Zentralafrikanischen Republik, in Syrien, im Südsudan und in Darfur - mangelt es schlicht an Kenntnis darüber, welche konkreten zivilen Maßnahmen die entscheidenden Krisentreiber beeinflussen könnten. Bei den Entwicklungen in Libyen und Syrien lässt sich trefflich darüber streiten, an welcher Stelle der Westen Fehler gemacht oder Chancen verpasst hat. Eines ist aber unstrittig: Das Spektrum an Handlungsalternativen, wie sie u.a. auch aus Kreisen der Friedensforschung und Zivilgesellschaft vorgeschlagen wurde, war immer sehr eng.

Frühwarnung versagt jedoch auch dann, wenn anderen Zielen als dem der Gewaltprävention Vorrang eingeräumt wird. Die europäischen Staaten reagierten auf die ersten Demonstrationen im Arabischen Frühling weniger aus der Motivation heraus, Gewalt zu verhindern, als vielmehr in der Absicht, die Demokratisierung dieser Länder zu unterstützen. Erst mit der gewaltsamen Zuspitzung infolge zunehmender staatlicher Repression passte auch der Westen sein Vorgehen an und setzte mehr auf Deeskalation.
Aber auch Zielkonflikte können den Erfolg von Frühwarnung beeinträchtigen: Erinnert sei hier an das Ergebnis der umstrittenen Wahlen in Kenia vom März 2013. Nachdem die Wahlkommission Uhuru Kenyatta mit einem denkbar knappen Vorsprung offiziell zum Sieger erklärt hatte, stand jedes Mitglied der Staatengemeinschaft vor der Alternative, entweder das Ergebnis formal anzuerkennen und dem neuen Präsidenten zu gratulieren - oder dieses nicht zu tun und damit die Legitimität des Wahlverfahrens öffentlich in Zweifel zu ziehen. Letzteres, darin waren sich die Analysten einig, hätte die bereits angespannte politische Lage zu einem äußerst kritischen Punkt eskaliert. Dementsprechend standen auch die Anhänger des unterlegenen Kandidaten vor einem Dilemma: Entweder gaben sie dem Recht auf faire demokratische Wahlen Priorität und versuchten Neuwahlen zu erzwingen, womit sie jedoch einen Rückfall in die Gewalt riskiert hätten. Oder sie akzeptierten um der Stabilität willen die Situation. Die kenianische Bevölkerung und die europäischen Regierungen entschieden sich für die Präventionsstrategie und erkannten das vom Verfassungsgericht bestätigte Wahlergebnis an. In solchen Situationen kann Frühwarnung Handlungsorientierung geben, aber die Entscheidung, welchem Ziel Vorrang eingeräumt wird, bleibt genuin politischer Natur.

Eine realistische Einschätzung der Grenzen und Möglichkeiten von Frühwarnung und Prävention muss akzeptieren, dass beides sehr wohl gelingen, aber auch scheitern kann. Dennoch müssen die Bemühungen im Bereich der zivilen Krisenprävention und der Friedensforschung weiter auf- und ausgebaut werden, um das erforderliche Wissen und damit die Chancen auf Erfolg zu verbessern.

Angelika Spelten ist wissenschaftliche Mitarbeiterin am Institut für Entwicklung und Frieden (INEF) und vertritt das Institut in der Arbeitsgemeinschaft Frieden und Entwicklung (FriEnt).

\section{Früherkennung und zivile Krisenprävention kommen im politischen Tagesgeschäft zu kurz}

\section{Winfried Nachtwei}

Im Koalitionsvertrag von 1998 verpflichteten Abgeordnete von SPD und Grünen erstmalig eine Bundesregierung auf die „Entwicklung und Anwendung von wirksamen Strategien und Instrumenten der Krisenprävention und der friedlichen Konfliktregelung“. Dahinter standen das friedenspolitische Desaster der Balkankriege, die selbstkritische Diskussion in der Entwicklungszusammenarbeit um ihre Krisenwirkungen und das Drängen der Friedens- und Konfliktforschung. Die ersten Umsetzungsschritte wie der Aufbau des Zivilen Friedensdienstes und des Zentrums Internationaler Friedenseinsätze, der Deutschen Stiftung Friedensforschung, die Förderung von Krisensensibilität in der Entwicklungszusammenarbeit machten Hoffnungen auf mehr. 2001 gelang es sogar, in Mazedonien den drohenden nächsten Balkankrieg zu verhüten - durch LastMinute-Früherkennung, konzertierte internationale Prävention und politische Einigung der Konfliktparteien. 
Die Blütezeit dauerte aber nicht lange. Als der Aktionsplan „Zivile Krisenprävention, Konfliktlösung und Friedenskonsolidierung" 2004 vom Bundeskabinett beschlossen wurde, hatte sich die Großwetterlage im Zuge des global war on terrorism längst verschoben. Schon im ersten Umsetzungsbericht (2006) der Bundesregierung erfuhr der Präventionsbegriff eine auffällige Entgrenzung und Verflachung: Es war nur noch von Konfliktnachsorge und Friedenskonsolidierung, kaum noch von operativer Primärprävention die Rede. Diese Tendenz verstärkte sich in den Umsetzungsberichten von 2008 und 2010. Das notorische Präventionshindernis - der blockierte Übergang von Early Warning zu Early Action - wurde nicht thematisiert. Zugleich wurden Militäreinsätze und -kapazitäten unterschiedslos unter den Begriff der Prävention subsumiert und damit der Begriff ziviler Krisenprävention entkernt.

Bei Stabilisierungseinsätzen zeigten sich alsbald Strukturmängel: Im Kosovo explodierte der vernachlässigte Konflikt 2004 in den Märzunruhen. In Afghanistan mehrten sich im Sommer 2006 auch im Norden die Alarmsignale. Die politische Führung in Berlin weigerte sich jedoch bis 2009, die sich verschärfende Lage zur Kenntnis zu nehmen. Die verpassten Chancen frühzeitiger Gegensteuerung legen den Schluss nahe, wirksamere Krisenprävention wäre möglich gewesen - ohne der Illusion zu verfallen, jede Eskalation erkennen, gar prognostizieren und rechtzeitig stoppen zu können. Dazu sind gesellschaftliche und politische Prozesse viel zu unberechenbar und von außen kaum zu beeinflussen.

Wie ist die deutsche Politik nun aufgestellt? Die Bundesregierung verfügt vor allem auf Ressortebene über verschiedene, teilweise sogar mit internationalen Frühwarnsystemen verbundene Instrumente der Krisenfrüherkennung. Ihre wichtigsten Sensoren sind die flächendeckend um den Globus verteilten Botschaften. Sie stehen fast täglich in Kontakt mit ihren Länderreferaten im Auswärtigen Amt (AA). Die Krisensensibilität der Außenvertretungen hängt aber nicht zuletzt von den jeweiligen Personen ab. Viel zu oft herrschte in der Vergangenheit ein Stabilitätsverständnis „von oben“ vor, das mit einer gewissen Distanz und damit Unkenntnis gegenüber lokalen Gesellschaften einherging. Ein Runderlass weist jedoch seit einigen Monaten die Botschaften an, im Rahmen ihrer Berichterstattung auch das Risiko von Massenverbrechen zu beachten und Maßnahmen zu ihrer Verhütung vorzuschlagen. Als Nadelöhr erweisen sich jedoch die Länderreferate, deren Abstand zur politischen Leitungsebene erheblich sein kann.

Zentrales Hilfsinstrument der mittelfristigen Vorausschau sind länderbezogene Analysen, die der Bundesnachrichtendienst (BND) unter Zuarbeit anderer Ressorts in einem Turnus von 12 bis 18 Monaten erstellt. Sie sollen die Konfliktstruktur eines Landes erfassen, auf Eskalationsmomente hinweisen und die politische Entscheidungsfindung unterstützen. Sie werden im „Krisenvorsorgeinformationssystem“ auch der Bundesregierung zur Verfügung gestellt.

Im Auftrag des Bundesministeriums für wirtschaftliche $\mathrm{Zu}$ sammenarbeit und Entwicklung (BMZ) erstellt das German Institute for Global and Area Studies (GIGA) jährlich eine Analyse zu innerstaatlicher Gewaltneigung, Konflikt- und Risikopotenzialen in Kooperationsländern. Der Fokus auf strukturelle und prozessuale Konfliktfaktoren eignet sich insbesondere für längerfristige Prävention. Als Basis kurzfristiger Früherkennung fungieren die zweimal im Monat erscheinenden, allerdings geheimen Indication \& Warning-Meldungen des BND.

Seit 2012 befasst sich in der Abteilung "Strategie und Einsatz" des Verteidigungsministeriums (BMVg) das Referat SI3 mit der Früherkennung für etwa 20 Krisenländer weltweit: Die Relevanz für Präventionsmaßnahmen leitet sich dann aber nicht nur aus der Lage, sondern auch aus dem Betroffenheitsgrad Deutschlands ab. Gegebenenfalls ergehen Empfehlungen an das BMVg, wenngleich die Federführung für Krisenprävention beim AA verbleibt. Dessen Krisenreaktionszentrum (KRZ), an das Fähigkeiten anderer Ressorts angekoppelt sind, ist für den Schutz deutscher Staatsbürger zuständig. Sein Repertoire umfasst Warnung und Prävention, aber auch Planung und Durchführung von Schutzmaßnahmen und Evakuierungsoperationen im Krisenfall. Ausgeklammert bleiben jedoch die allgemeine Konfliktfrüherkennung zum Zweck politischer Krisenprävention und der Verhütung von Massenverbrechen gemäß der internationalen Schutzverantwortung (R2P). Für diese gibt es in der Bundesregierung keinen zentralen Ort. Der Ressortkreis „Zivile Krisenprävention“ sollte zwar laut Aktionsplan „Schnittstelle zur Überführung von Early Warning [...] in Early Action“ sein, allerdings konnte er diese Funktion mangels Kapazitäten und fehlender Anbindung an die politische Leitung nie ausfüllen. Beim R2P-Focal Point, der seit 2012 beim Leiter der AA-Abteilung „Vereinte Nationen und Globale Fragen" besteht, handelt es sich bislang nur um eine zusätzliche Zuständigkeit ohne Unterbau. Frühwarninstrumente einzelner Ressorts blieben gleichsam Feuermelder ohne Leitstelle.

Der Bundestag spielt in der deutschen Außenpolitik, die laut Grundgesetz Friedenspolitik sein soll, eine relativ starke Rolle. Die Initiativen zur zivilen Krisenprävention kamen aus seinen Reihen. Haushaltsrecht und Parlamentsbeteiligung bei Auslandseinsätzen der Bundeswehr eröffnen Einflusschancen. Fachpolitiker einschlägiger Ausschüsse (z.B. Auswärtiges, Entwicklung) beobachten und bereisen auch Krisenländer, nehmen an internationalen Parlamentarierversammlungen teil und fungieren bisweilen als Wahlbeobachter. Dieses Früherkennungsund Präventionspotenzial kommt aber nur punktuell, nicht systematisch zum Tragen. Die Zwänge des Abgeordnetenalltags zwischen Ausschussarbeit, Fraktionssitzungen, Außenterminen und Wahlkreisverpflichtungen begünstigen darüber hinaus die Sorge um die Überforderung eigener Kapazitäten und damit eine Abwehrhaltung gegenüber Zusatzaufgaben. Aufgrund der Ferne vieler Fachpolitiker zum Machtzentrum verhallen ihre Appelle oft schon im Plenum des Bundestags. Trotzdem können Abgeordnete in Einzelfällen wichtige Impulse zur Früherkennung und Prävention setzen, indem sie die Aufmerksamkeit für Krisenprozesse verstärken. Außergewöhnlich war das konzertierte Präventionsengagement des Unterausschusses „Zivile Krisenprävention und Vernetzte Sicherheit“, des AA und der internationalen Organisationen im Vorfeld der Unabhängigkeit des Südsudan 2011.

Welche politischen Konsequenzen lassen sich ableiten? Die neue Große Koalition verspricht im Koalitionsvertrag, Strukturen ziviler Krisenprävention „stärken und weiterentwickeln“ zu wollen. Außenminister Frank-Walter Steinmeier und Bundes- 
präsident Joachim Gauck betonten bei der jüngsten Münchner Sicherheitskonferenz, Deutschland müsse mehr internationale Verantwortung übernehmen und sich „früher, entschiedener und substanzieller einbringen“. Gauck begrüßte ausdrücklich, dass die Bundesregierung bei der Schutzverantwortung „besonders auf Prävention, auf internationale Zusammenarbeit sowie auf die Entwicklung von Frühwarnsystemen gegen Massenverbrechen setzt". Dazu bedarf es neben einer professionellen Öffentlichkeitsarbeit dringend der Weiterentwicklung nationaler Fähigkeiten:

- Elementar bleibt der politische Wille zur operativen Krisenprävention insbesondere zur Verhütung von Massenverbrechen. Das verlangt einen Mentalitätswandel: „Fahren auf Sicht“ ist immer mal wieder unvermeidbar, reicht aber nicht aus. Wir brauchen mehr Klarheit über Voraussetzungen und Hemmnisse, Chancen und Grenzen von Früherkennung und Prävention - gerade angesichts globalisierter Konfliktdynamiken sowie abnehmender Steuerungsfähigkeit der Politik.

- Die Schlüsselfrage lautet: Wie kommen die richtigen Informationen zur rechten Zeit an die richtigen Personen? Dazu müssten Erkenntnisse der Ressorts beim AA möglichst nahe am KRZ zusammengeführt und bewertet werden. Ein Fokus sollte dabei auf der Verhütung von Massenverbrechen im Sinne der R2P liegen. Das erfordert eine enge Zusammenarbeit mit den Frühwarnsystemen von UN und Regionalorganisationen.
- Dort, wo es vorrangig um die lokale Lösung lokaler Konflikte geht, geben „bodennahe“ Regionalexpertise, bei schwersten Menschenrechtsverbrechen auch direkte Meldekanäle sowie die Unterstützung lokaler und regionaler Frühwarnsysteme den Ausschlag, nicht so sehr standardisierte Frühwarnsysteme mit ihren Indikatoren, Matrixen und Simulationsmodellen.

- Um nicht im Early Warning stecken zu bleiben, gilt es, in den Krisenländern zu stärkende Struktur- und Prozessfaktoren sowie präventive Handlungsoptionen herauszuarbeiten. Eine enge Anbindung an die politische Leitung ist existentiell.

- Bundestag und zivilgesellschaftliche Akteure bleiben für präventive Politik unverzichtbar. Daher sind sie in geeigneter Weise in die systematische Früherkennung einzubeziehen. Als Orte böten sich der Unterausschuss des Bundestags und der Beirat Zivile Krisenprävention an.

In seiner Münchner Rede fragte Gauck: „Engagieren wir uns schon ausreichend dort, wo die Bundesrepublik eigene und eigens Kompetenz entwickelt hat - nämlich bei der Prävention von Konflikten?“ Die Antwort lautet: „Eindeutig nein!“ Die ersten zehn Jahre des Aktionsplans zeigen: Ohne einen Zuwachs an politischem Umsetzungswillen und Ressourcen bleibt Prävention eher wohlfeile Rhetorik als politische Praxis.

Winfried Nachtwei war für Bündnis 90/Die Grünen von 1994 bis 2009 Mitglied des Deutschen Bundestages und ist Ko-Vorsitzender des Beirats Zivile Krisenprävention beim Auswärtigen Amt.

\section{NEUERSCHEINUNGEN}

\section{Ute Runge}

\section{Themenschwerpunkt - Special focus topic}

Interventionen zwischen Krieg und Ethik. Das Beispiel Mali Interventions between War and Ethics. The Example of Mali

Ammour, Laurence Aïda/Berghezan, Georges/ Bouhlel, Ferdaous/Deycard, Frédéric/ Grémont, Charles/ Rouppert, Bérangère/ Tisseron, Antonin: Sahel. Éclairer le passé pour mieux dessiner l'avenier, Bruxelles (GRIP) 2013.

Flichy, Thomas (Hrsg.): Opération Serval au Mali. L'intervention française décryptée, Panazol (Lavauzelle) 2013.

Frémeaux, Jacques/ Evanno, Philippe/ Chauprade Aymeric (Hrsg.): Menaces en
Afrique du Nord et au Sahel et sécurité globale de l'Europe, Paris (Ellipses) 2013.

Galy, Michel (Hrsg.): La guerre au Mali. Comprendre la crise au Sahel et au Sahara. Enjeux et zones d'ombre, Paris (La Découverte) 2013.

Lasserre, Isabelle/ Oberlé, Thierry: Notre guerre secrète au Mali. Le nouvelles menaces contre la France, Paris (Fayard) 2013.

\section{Theorien internationaler Beziehungen - International relations theory}

Antonczy, Eric/ Feske, Susanne/ Oerding, Simon (Hrsg.): Einführung in die Internationalen Beziehungen. Ein Lehrbuch, Leverkusen (Budrich) 2014.

Cesa, Marco (Hrsg.): Machiavelli on International Relations, Oxford (Oxford University Press) 2014.
Masala, Carlo: Kenneth N. Waltz. Einführung in seine Theorie und Auseinandersetzung mit seinen Kritikern, Baden-Baden (Nomos Verlagsgesellschaft) 2014.

Outhwaite, William: Critical Theory and Contemporary Europe, New York, NY (Continuum Publishing) 2014.

\section{Völkerrecht und internationale Organisationen - International law and organisations}

Bosco, David L.: Rough Justice: The International Criminal Court in a World of Power Politics, Oxford (Oxford University Press) 2014.

Chartier, Gary: Radicalizing Rawls. Global Justice and the Foundations of International Law, Basingstoke (Palgrave Macmillan) 2014. 